\title{
Pulsating flow and convective heat transfer in a cavity with inlet and outlet sections
}

\author{
A. Velazquez , J.R. Arias, J.L. Montanes \\ Aerospace Propulsion and Fluid Dynamics Department, School of Aeronautics, Universidad Politecnica de Madrid, Plaza del Cardenal Cisneros 3, 28040 Madrid, Spain
}

A R T I C L E I N F O

Keywords:

Cavity

Unsteady flow

Convective heat transfer

\begin{abstract}
A B S T R A C T
This paper deals with the study of 2-D, laminar, pulsating flow inside a heated rectangular cavity with different aspect ratios. The cooling liquid (water with temperature dependent viscosity and thermal conductivity) comes and leaves the cavity via inlet and outlet ports. The flow topology is characterised by the large recirculation regions that exist at inner corners of the cavity. These low velocity regions cause the heat transfer to be small when compared, for instance, to that of a straight channel. We study the effect that a prescribed pulsation at the inlet port has on the cavity heat transfer. This pulsating boundary condition, of the unsteady Poiseuille type, is described by its frequency and the amplitude of the pressure gradient. The time averaged Reynolds number of the flow, based on the hydraulic diameter of the inlet channel, is 100 and we consider that the dimensionless pulsation frequency (Strouhal number) varies in the range from 0.0 to 0.4 . We show that the prescribed pulsation enhances heat transfer in the cavity and that the mechanism that causes this enhancement appears to be the periodic change in the recirculation flow pattern generated by the pulsation. Regarding the quantitative extent of heat transfer recovery, we find that appropriate selection of the pulsation parameters allows for the cavity to behave like a straight channel that is the configuration with the highest Nusselt number.
\end{abstract}

\section{Introduction}

Natural, forced or mixed convection inside rectangular enclosures is a subject of practical interest in quite a number of industrial applications. These applications are as diverse as, for instance, design of food processing systems, MEMS manufacturing, or heat exchanger optimisation. Because of this fact, a large number of papers have been recently published on this specific subject in the specialised literature. Also, a cavity with non-aligned inlets and outlet ports could be seen as an abrupt deviation in an otherwise straight channel. This situation appears, for example, when designing integrated cooling systems for electronics applications. If the micro-cooling system is merely placed on top of the electronics components, the micro-channels that make up the micro-heat exchanger are normally straight. However, if the goal is to come up with an integrated setup and the micro-channels are to be placed inside the electronics hardware itself, it is possible that they cannot be kept straight because of a number of product design constrains.

The natural convection regime inside a cavity has been analysed extensively. Two of the most recent articles that deal with this situation, and that include a heated body inside the enclosure, are those of Angeli et al. [1] and Kim et al. [2]. The case of an adiabatic body located inside the enclosure has been addressed by $\mathrm{Ha}$ et al.
[3]. In these three references, the authors analysed the flow topology and its influence on heat transfer. Also, they provided local information on the Nusselt number at the enclosure walls and in the case of reference [1] they summarised the information in the shape of general correlations. Unsteady periodic natural convection in a square cavity with no inner body has been studied by Kalabin et al. [4]. Mixed convection inside a square enclosure with an internal partition has been addressed by Mahapatra et al. [5]. The influence of different aspect ratios of the cavity has been analysed by Guo and Sharif [6].

In the references mentioned above, the cavities were closed. A different type of problem appears whenever inlet and outlet ports are present. Saeidi and Khodadadi [7] have analysed the steady laminar forced convection inside a square cavity for different relative positions of the inlet and outlet openings. They studied the flow topologies in the range of Reynolds numbers from 10 to 500 (based on the hydraulic diameter of the inlet channel) and searched for configurations that maximise heat transfer while minimising pressure drop. This is important in practical engineering applications because the pressure drop is related to the cost to operate the system. For example, this pressure drop is important, as the author points out, in the design of microfilters [8] and piezoelectric microvalves [9]. Pulsating flow in a heated cavity has been recently studied, also, by Saeidi and Khodadadi [10]. In this paper, the authors consider an oscillating laminar flow inside a fixed geometry enclosure. In particular, inlet and outlet port sections have a width that is $15 \%$ of the side wall length. In practice, this 


\begin{tabular}{|c|c|c|c|}
\hline \multicolumn{4}{|c|}{ Nomenclature } \\
\hline$A$ & inlet and outlet channels height & $t^{\prime}$ & time \\
\hline$a_{2}$ & control parameter for pressure gradient at inlet section & $t^{*}$ & dimensionless pseudo-time \\
\hline$B$ & step height & $T$ & dimensionless fluid temperature \\
\hline C & cavity length & $T^{\prime}$ & temperature \\
\hline$C_{p \_ \text {inlet }}$ & specific heat at inlet section & $T_{\text {inlet }}$ & fluid temperature at inlet section \\
\hline & computational time step & $T_{\text {wall }}$ & temperature at cavity walls \\
\hline$D$ & $\begin{array}{l}\text { length of the heated portion downstream of the outlet } \\
\text { port }\end{array}$ & $T_{\text {wall }+1}$ & $\begin{array}{l}\text { temperature of the fluid in the grid point closest to cav- } \\
\text { ity walls }\end{array}$ \\
\hline$D_{\mathrm{h}}$ & hydraulic diameter of the inlet channel & $u$ & dimensionless horizontal velocity component \\
\hline $\mathrm{Gr}$ & Grashof number & $u^{\prime}$ & horizontal velocity component \\
\hline$k$ & dimensionless thermal conductivity & $u_{\text {inlet }}$ & mean horizontal velocity component at inlet section \\
\hline$k^{\prime}$ & thermal conductivity & $v$ & dimensionless vertical velocity component \\
\hline$k_{\text {inlet }}$ & thermal conductivity at inlet section & $v^{\prime}$ & vertical velocity component \\
\hline$k_{\text {wall }}$ & thermal conductivity at wall temperature & $x$ & dimensionless horizontal co-ordinate \\
\hline$n$ & direction normal to a surface & $x^{\prime}$ & horizontal co-ordinate \\
\hline$P$ & dimensionless pressure & $y$ & dimensionless vertical co-ordinate \\
\hline$P$ & pressure & $y^{\prime}$ & vertical co-ordinate \\
\hline $\mathrm{Pr}$ & Prandtl number & & \\
\hline$Q_{C}$ & normalised heat transfer in the cavity & \multicolumn{2}{|c|}{ Greek symbols } \\
\hline$Q_{0}$ & $\begin{array}{l}\text { normalised heat transfer in the heated portion down- } \\
\text { stream of outlet port }\end{array}$ & $\begin{array}{l}\beta \\
\delta^{\prime}\end{array}$ & $\begin{array}{l}\text { pseudo-compressibility parameter } \\
\text { Stokes layer thickness }\end{array}$ \\
\hline$Q_{i}^{\prime}$ & dimensional heat transfer per unit of span element ' $i$ ' & $\Delta$ & increment \\
\hline$Q_{\text {ref }}$ & $\begin{array}{l}\text { dimensionless heat transfer in the reference straight } \\
\text { channel }\end{array}$ & $\begin{array}{l}\Delta_{\|} \\
\Delta_{\perp}\end{array}$ & $\begin{array}{l}\text { grid spacing parallel to the wall } \\
\text { grid spacing perpendicular to the wall }\end{array}$ \\
\hline$Q_{\mathrm{T}}^{\prime}$ & dimensional heat transfer in a whole wall & $\mu$ & dimensionless dynamics viscosity \\
\hline$Q_{\mathrm{T}}$ & $\begin{array}{l}\text { normalised heat transfer in the cavity plus a down- } \\
\text { stream portion }\end{array}$ & $\begin{array}{l}\mu^{\prime} \\
\mu_{\text {inlet }}\end{array}$ & $\begin{array}{l}\text { dynamic viscosity } \\
\text { dynamic viscosity at inlet section }\end{array}$ \\
\hline $\operatorname{Re}$ & Reynolds number based on $D_{\mathrm{h}}$ and $u_{\infty}$ & $v^{\prime}$ & kinematic viscosity \\
\hline $\operatorname{Re}_{\delta^{\prime}}$ & Reynolds number based on the Stokes thickness & $\rho_{\text {inlet }}$ & density at inlet section \\
\hline St & Strouhal number & $\omega$ & dimensionless pulsation frequency \\
\hline$t$ & dimensionless time & $\omega^{\prime}$ & dimensional pulsation frequency \\
\hline
\end{tabular}

means that they have a flow topology characterised by a recirculation region that fills in most of the cavity volume. For this geometry, the authors performed a series of computations covering a rather wide range of Strouhal numbers (from 0.1 to 10 ) and they report that, in all cases, heat transfer is enhanced when inlet flow oscillation is enforced. Steady mixed convection inside a cavity where a heated rectangular cylinder is present has been analysed by Shuja et al. [11]. In particular, these authors focussed on the influence of the heated body size and the position of the exit port. Steady mixed convection in a rectangular cavity with differentially heated walls and inlet and outlet sections was studied by Singh and Sharif [12]. In this paper, the authors gave indications regarding the most efficient configuration from the heat transfer point of view. A practical application of the study of forced convection in a rectangular cavity has been presented by Verboven et al. [13] where they study the airflow and heat transfer inside a microwave oven and their influence on the quality of the cooked material. Finally, it is to be noted that heat transfer inside a cavity has also been studied in the context of heating/cooling of a near critical fluid [14] and in connection to the use of acoustic waves to enhance thermal effects $[15,16]$.

In the present paper, we consider the problem of a pulsating laminar flow inside a heated cavity that has inlet and exit ports. Our working fluid is water with temperature dependent viscosity and thermal conductivity. The reason is that, when planning for a future experimental campaign, it is necessary to account for the fact that water viscosity changes by a factor of 3 in the range from 20 to $80 \mathrm{C}$. We study heat transfer as a function of the pulsation frequency and aspect ratio of the cavity. When compared to a straight channel, a cavity is characterised by the fact that large flow recirculation regions appear at the corners and that heat transfer degrades in these regions. The questions that we ask in this paper are: (a) is it possible to recover the high heat transfer rates of the straight channel by pulsating the flow that comes into the cavity? (b) up to what extent is this recovery possible?, and (c) what is its dependency on the enclosure aspect ratio and pulsation frequency? Regarding paper organisation, the problem description is addressed in the next section; problem formulation and numerical methodology come next and, then, results and conclusions are presented.

\section{Problem description}

We consider the unsteady laminar flow of water in a 2D cavity with inlet and outlet ports, see Fig. 1 . The height $A$ of both inlet and outlet channels is the same and we consider different values of the step height $(B)$ and horizontal length of the cavity (C). In particular we study eight possible geometry cross-combinations: $B=0.0,0.5 A, 1.0 A$ and $1.5 A$, and $C=2.0 A$, and $4.0 A$. This causes the cavity aspect ratio to change in the range from 0.80 up to 2.66. The cases with $B=0.0$ represent a straight channel and are used as a baseline for comparison purposes. We define the flow Reynolds number $\operatorname{Re}$ as: $\rho_{\text {inlet }} u_{\text {inlet }} D_{\mathrm{h}} / \mu_{\text {inlet }}$, where $\rho_{\text {inlet }}$, $u_{\text {inlet }}, D_{\mathrm{h}}$ and $\mu_{\text {inlet }}$ are inlet section density, average velocity, hydraulic diameter and viscosity, respectively. We consider the case of $R e=100$ that could be practically achieved (for inlet temperature equal to $293 \mathrm{~K}$ ) with an inlet height of $500 \mu \mathrm{m}$ and an inlet velocity of $0.1 \mathrm{~m} / \mathrm{s}$. The cavity walls (see Fig. 1) have constant temperature of $353 \mathrm{~K}$. A short portion $D=C$ downstream of the outlet port is also heated. Walls are adiabatic upstream of section a-e and downstream of section d-i (see Fig. 1). Because of the dependency of water transport coefficients with 


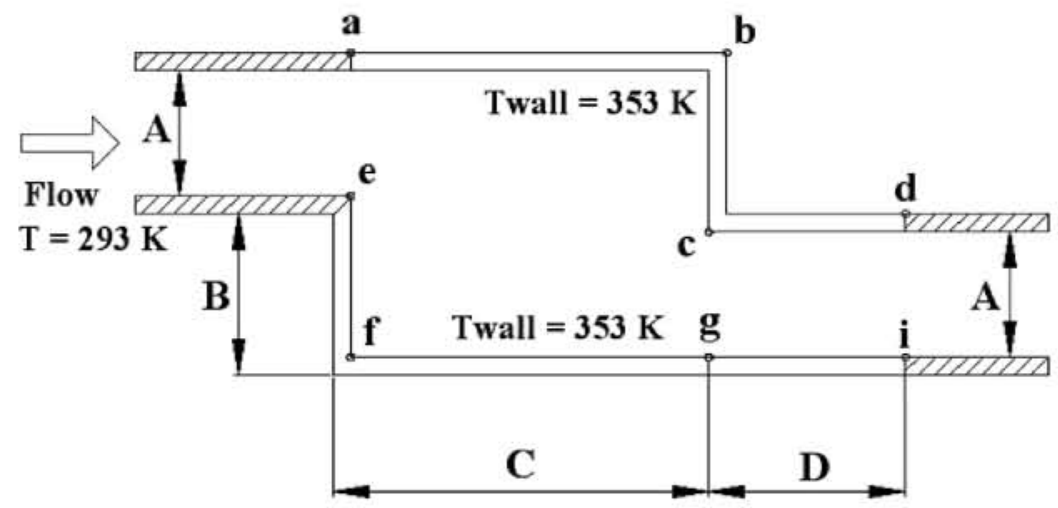

Fig. 1. Overview of the generic configuration of the problem.

temperature in the range of 293-353 K, we assume temperature dependent viscosity and thermal conductivity.

To simulate pulsating conditions, we prescribe an unsteady Poiseuille solution at the entrance of the inlet channel. This solution is described by two parameters: the pulsation frequency $\omega^{\prime}$ and amplitude of the pressure gradient. In this study we have selected the largest amplitude that avoids flow reversal at the end of the outlet channel. Regarding frequency, we considered (for each of the eight different geometry cases) six different dimensionless pulsation frequencies $\omega=\omega^{\prime} D_{\mathrm{h}} / u_{\text {inlet }}$. These dimensionless frequencies could be regarded as the Strouhal number of the problem. The different values of the frequencies were: $\omega=0.0,0.10$, $0.15,0.20,0.30$ and 0.40 . That is, we covered the range from the steady case $(\omega=0)$ to the moderately unsteady regime $(\omega=0.4)$. For all cases we computed the heat transfer and compared the results obtained with different combinations of parameters.

\section{Problem formulation and solution procedure}

The equations of the problem are the 2D unsteady laminar Navier-Stokes equations with temperature dependent viscosity and thermal conductivity. In dimensionless form, these equations are:

$\frac{\partial u}{\partial x}+\frac{\partial v}{\partial y}=0$

$\frac{\partial u}{\partial t}+u \frac{\partial u}{\partial x}+v \frac{\partial u}{\partial y}=-\frac{\partial P}{\partial x}+\frac{1}{R e}\left[\mu\left(\frac{\partial^{2} u}{\partial x^{2}}+\frac{\partial^{2} v}{\partial y^{2}}\right)+2 \frac{\partial \mu}{\partial x} \frac{\partial u}{\partial x}+\frac{\partial \mu}{\partial y}\left(\frac{\partial u}{\partial y}+\frac{\partial v}{\partial x}\right)\right]$

$\frac{\partial v}{\partial t}+u \frac{\partial v}{\partial x}+v \frac{\partial v}{\partial y}=-\frac{\partial P}{\partial y}+\frac{1}{R e}\left[\mu\left(\frac{\partial^{2} v}{\partial x^{2}}+\frac{\partial^{2} v}{\partial y^{2}}\right)+2 \frac{\partial \mu}{\partial y} \frac{\partial v}{\partial y}+\frac{\partial \mu}{\partial x}\left(\frac{\partial v}{\partial x}+\frac{\partial u}{\partial y}\right)\right]$

$\frac{\partial T}{\partial t}+u \frac{\partial T}{\partial x}+v \frac{\partial T}{\partial y}=\frac{1}{\operatorname{RePr}}\left[k\left(\frac{\partial^{2} T}{\partial x^{2}}+\frac{\partial^{2} T}{\partial y^{2}}\right)+\frac{\partial k}{\partial x} \frac{\partial T}{\partial x}+\frac{\partial k}{\partial y} \frac{\partial T}{\partial y}\right]$

Dimensionless variables are defined as:

$u=\frac{u^{\prime}}{u_{\text {inlet }}}, \quad v=\frac{v^{\prime}}{u_{\text {inlet }}}, \quad P=\frac{P^{\prime}}{\rho_{\text {inlet }} u_{\text {inlet }}^{2}}, \quad T=\frac{T^{\prime}}{T_{\text {inlet }}}$

$\chi=\frac{\chi^{\prime}}{D_{\mathrm{h}}}, \quad y=\frac{y^{\prime}}{D_{\mathrm{h}}}, \quad t=\frac{t^{\prime} u_{\text {inlet }}}{D_{\mathrm{h}}}$

where 'prime' denotes the dimensional variables. $T_{\infty}$ is the temperature at the entrance section of the inlet channel. We have assumed that viscosity and thermal conductivity depend on temperature as a second-order polynomial. The coefficients of the polynomials have been adjusted via least squares by using the experimental data provided by Incropera and DeWitt [17]. The functional correlations are:

$$
\begin{aligned}
& \mu=\frac{\mu^{\prime}}{\mu_{293 \mathrm{~K}}^{\prime}}=1-5.646 \cdot(T-1)+12.259 \cdot(T-1)^{2} \\
& k=\frac{k^{\prime}}{k_{293 \mathrm{~K}}^{\prime}}=1+0.786 \cdot(T-1)-1.176 \cdot(T-1)^{2}
\end{aligned}
$$

With regard to the boundary conditions, we have followed the formulation used previously [18] in our study of the flow past a backwards facing step. In particular, the inflow boundary condition for the horizontal velocity is the unsteady Poiseuille flow:

$u(y, t)=u_{1}(y)+u_{2}(y, t)$

where $u_{1}(y)$ and $u_{2}(y, t)$ are defined by the following relations:

$u_{1}(y)=-24\left(y^{2}-\frac{y}{2}\right)$

$u_{2}(y, t)=\operatorname{Real}\left[\frac{\mathrm{i} a_{1} a_{2} \mathrm{e}^{\mathrm{i} 2 \pi \omega t}}{2 \pi \omega}\left(\frac{1}{\alpha+1} \mathrm{e}^{\psi y}+\frac{\alpha}{\alpha+1} \mathrm{e}^{-\psi y y}-1\right)\right]$

with

$a_{1}=\frac{48}{\operatorname{Re}}, \quad \psi=(\mathrm{i} 2 \pi \omega R e)^{1 / 2}, \quad \alpha=\mathrm{e}^{\psi / 2}$

And for the remaining variables:

$v=0$

$\frac{\partial^{2} P}{\partial x^{2}}=0$

$T=1$

The outflow boundary conditions are:

$\frac{\partial u}{\partial x}=\frac{\partial v}{\partial x}=\frac{\partial^{2} P}{\partial x^{2}}=\frac{\partial T}{\partial x}=0$

Finally, at solid walls we have:

$u=v=0$

Either $T=T_{\text {wall }}$ or $\frac{\partial T}{\partial n}=0$

Pressure at the walls is extrapolated form the interior by using a discretised version of the momentum equations with one sided derivatives.

In the model described above, we have assumed laminar behaviour even though the flow is pulsating. Laminar turbulent transition in these types of flows in straight channels has been studied 
by Das and Arakeri [19], Akhavan et al. [20,21] and Hino et al. [22]. They provided transition criteria based on the Reynolds number defined by using the Stokes layer thickness: $\delta^{\prime}=\left(2 v^{\prime} / \omega^{\prime}\right)^{1 / 2}$, where $v^{\prime}$ is the kinematics viscosity, as the characteristic length. The transition threshold value is in the range from 500 to 1200 depending on the author. In our case, for the practical application described in the previous section, we have Reynolds numbers based on the Stokes layer thickness that are of the order of 50 , well below the above stated transition value. It is clear, of course, that the criterion reported in references [19-22] refers to a straight channel and it is not directly applicable to a cavity. However, given the fact that we are more than one-order of magnitude below the threshold, we believe that we could still assume laminar flow. Also, we did not include buoyancy terms in out model. The reason is that these terms scale as $G r / R e^{2}$, where $G r$ is the Grashof number, and are of the order of $1.0 \mathrm{e}-4$ in our case.

Regarding the inflow boundary conditions (9)-(14) we have chosen a functional behaviour (the unsteady Poiseuille flow) that is solution of the Navier-Stokes equations. This selection has the advantage of leading to a well posed problem. For example, if the inlet flow profiles do not fulfil the Navier-Stokes equations, algorithm stability problems may arise (which may require larger values of the artificial viscosity terms) and, in any case, a longer inlet channel computational domain may have to be selected so as to be sure that the flow entering the cavity does indeed fulfil the NavierStokes equations. When thinking of a practical application, it is not possible to choose the inlet boundary condition a priori because, in this situation, the pulsating pump (most probably of a reciprocating nature with two pistons because of cost, robustness and availability) becomes part of the flow system and the fluid variables at the cavity inlet port depend on the specific pump characteristics. Therefore, since we are studying a physical effect, we have selected a configuration that is independent of the pump and consistent with the basic equations. What we expect, but this will have to be assessed in a future experimental work, is that the qualitative engineering behaviour of the system does not depend very much on the details of the pulsating pump.

Concerning the solution procedure, an overview of the computational domain is presented in Fig. 2. As it has been described before, spatial co-ordinates are rendered dimensionless by using the hydraulic diameter of the inlet channel (twice the channel height) as the reference length of the problem. The numerical algorithm follows a finite point set approach for computation of the spatial derivatives as described in references $[18,23]$. This formulation leads to second-order accuracy in space. We used a Cartesian grid with spacing equal to 0.02 for both $x$ - and $y$-coordinates all over the computational domain. We had 21,438 points in the computational domain with the smallest cavity and 25,863 points in the one with the largest cavity. The use of Cartesian grids is not very efficient from the computational standpoint, and grid clustering should be used for practical industrial applications. However, since we focussed in this study on the physics aspects, we have opted for a robust, if not very efficient, computational approach. Continuity equation is treated by using a pseudo-compressibility formulation [24] with dual time $t^{*}$ integration. A description of the actual system of numerical equations being solved, including the physical time $t$ and

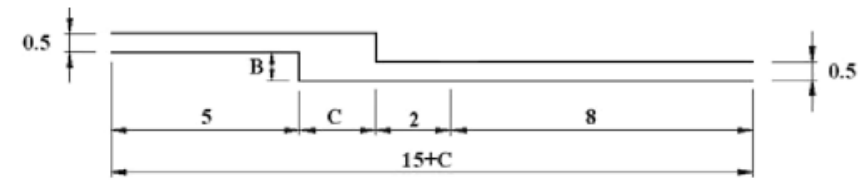

Fig. 2. Outline of the computational domain. the pseudo-time $t^{*}$, is provided in Eqs. (26)-(29) of reference [18]. In those equations, $\beta$ is the pseudo-compressibility parameter. A semi-implicit dual time Runge-Kutta approach has been followed for the nested required computational time integration. Details of this formulation are given in Eqs. (30) and (31) of reference [18]. Artificial dissipation terms are used to stabilise the convergence process. In particular, second-order Laplacian and fourth-order bi-Laplacian pressure terms are used for the continuity equation, and fourth-order bi-Laplacian velocity and temperature terms are used in the moment and energy equations, respectively. With this approach, time integration has a second-order accuracy.

\section{Results}

We have computed 48 cases that correspond to the following combination of parameters (see Fig. 1):

- $\operatorname{Re}=100 ; \operatorname{Pr}=6.62$.

- $T^{7}=293 \mathrm{~K}$ at inlet $(T=1)$.

- $T^{\prime}=353 \mathrm{~K}$ at walls a-b-c-d and e-f-g-i $(T=1.2)$.

- $A=0.5$.

- $B=0.0,0.5 A, 1.0 A, 1.5 A$.

- $C=2.0 A, 4.0 A$.

- $D=C$.

- $a_{2}=1.5 ; \omega=0.00,0.10,0.15,0.20,0.30,0.40$.

The time step and artificial compressibility parameter were $\mathrm{d} t=2.5 \mathrm{e}-4$ and $\beta=200$, respectively. Second- and fourth-order artificial dissipation parameters, see [18], were taken as $2.5 e-4$ and $1.0 \mathrm{e}-3$, respectively.

We started by computing the reference cases that correspond to the straight channel $(B=0)$, and we found that, in these cases, pulsation does not affect the time averaged heat transfer. This result is in agreement with the previous findings of Yu et al. [25] and Chattopadhyay et al. [26].

The dimensional heat transfer $Q^{\prime}$ per unit of span at any element ' $i$ ' of the walls is:

$Q_{i}^{\prime}=-K_{\text {wall }} \Delta_{\|} \frac{T_{\text {wall }+1}^{\prime}-T_{\text {wall }}^{\prime}}{\Delta_{\perp}}$

where $\Delta_{\|}$and $\Delta_{\perp}$ are horizontal and perpendicular grid spacing at the wall (that are the same in our simulation), and the subscript 'wall +1 ' stands for the grid point next to the wall. The previous expression, written in dimensionless variables and extended to the " $m$ " wall points reads as:

$Q=\frac{Q^{\prime}}{K_{\text {wall }} T_{\infty}}=\sum_{i=1}^{m}\left(T_{\text {wall }}-T_{\text {wall }+1}\right)$

Normalised heat transfer, $Q_{c}$, in the cavity (wall segments a-b-c and $\mathrm{e}-\mathrm{f}-\mathrm{g}$ in Fig. 1) as a function of the pulsation frequency for different values of the step height is presented in Fig. 3. Normalization has been achieved by dividing the heat transfer values computed by using expression (20) by the reference heat transfer value, $Q_{\text {ref, }}$ of the straight channel $(B=0)$. This figure shows that, in the steady case, the higher the step the lower the heat transfer in the cavity. In fact, a step height equal to one half of the inlet channel causes the heat transfer to drop by a factor of $25 \%$. When the step height is equal to the inlet channel height, steady heat transfer in the cavity degrades by a factor of $30 \%$. Further increments in the step height do not seem to degrade heat transfer more significantly.

An impression of the extent of the steady-state recirculation regions in the cavity for the case with $B=0.75 A$ is presented in Fig. 4. The situation changes when pulsation is prescribed at the entrance section of the inlet channel. Looking, again, at Fig. 3 it could 


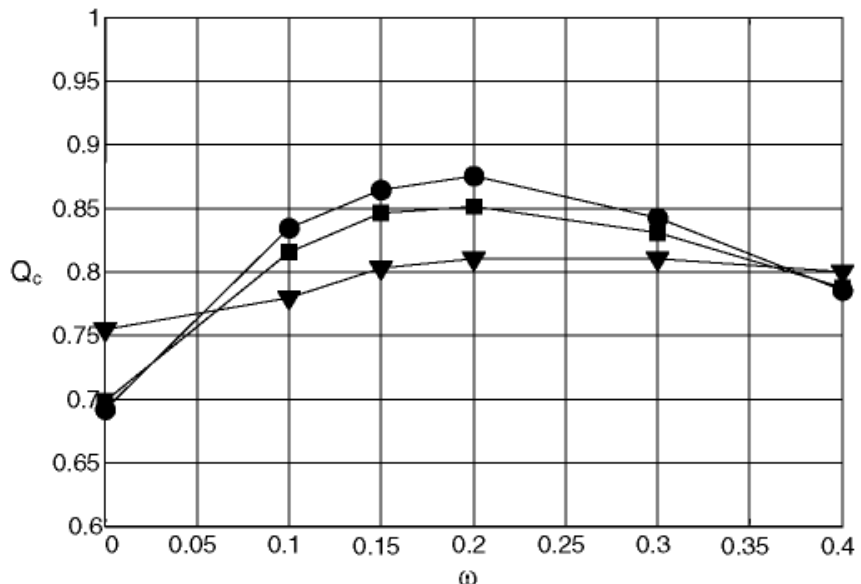

Fig. 3. Normalised cavity heat transfer as a function of the dimensionless pulsation frequency. $\boldsymbol{\nabla} B=0.5 A, \boldsymbol{\square} B=1.0 A$, and $B=1.5 A, C=4.0 A$.

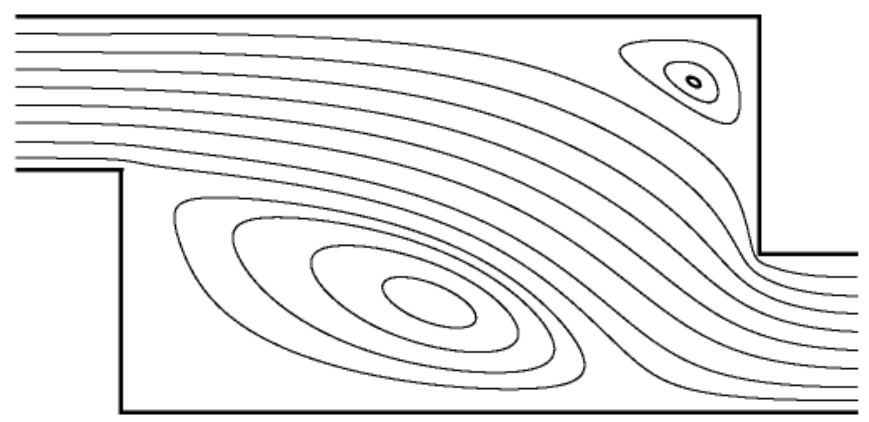

Fig. 4. Streamlines in the cavity in the steady-state case.

be seen that heat transfer increases as a function of the dimensionless frequency with a resonant behaviour. That is, heat transfer increases up to a certain frequency $(\omega=0.2)$ and decreases afterwards. Also, a distinctive feature in this case is that the higher the step height the more favourable the effect of pulsation. An evenly spaced sequence of streamlines fields along a cycle for the case of $\omega=0.2$ is presented in Fig. 5. In this figure, time reads top to bottom and left to right. In top right corner of the cavity, a recirculation region appears, grows, disappears, and appears again as a function of the time along the cycle. In the bottom left corner, the flow topology is more complex and two bubbles appear and disappear sequentially. It is to be noted that at some time instants, the two recirculation regions fill in more $50 \%$ of the cavity volume. The growth and decay of these regions also cause the main flow to change direction locally so it suggests the possibility of using this mechanism for flow control purposes. In this particular case, heat transfer in the cavity is recovered to the $88 \%$ level of the straight channel case when compared to the $69 \%$ level of the steady situation.

We now compute the normalised heat transfer in the cavity plus the additional wall portions $c-d$ and $g-i, Q_{T}$ (see Fig. 1 ). The results presented in Fig. 6 show that at $\omega=0.2$ it is possible to recover the heat transfer level of the straight channel. Also in this case it is apparent that cases with larger step height are more sensitive to the pulsation effects. The case with $B=A$ has also been computed by using the ideal fluid assumption (constant viscosity and thermal conductivity) so as to be certain that the behaviour been described does not depend on the selection of fluid properties. The results obtained are represented in this Fig. 6 by means of using a dashed line. It could be observed that the qualitative behaviour is similar to the one obtained by using real fluid proper-
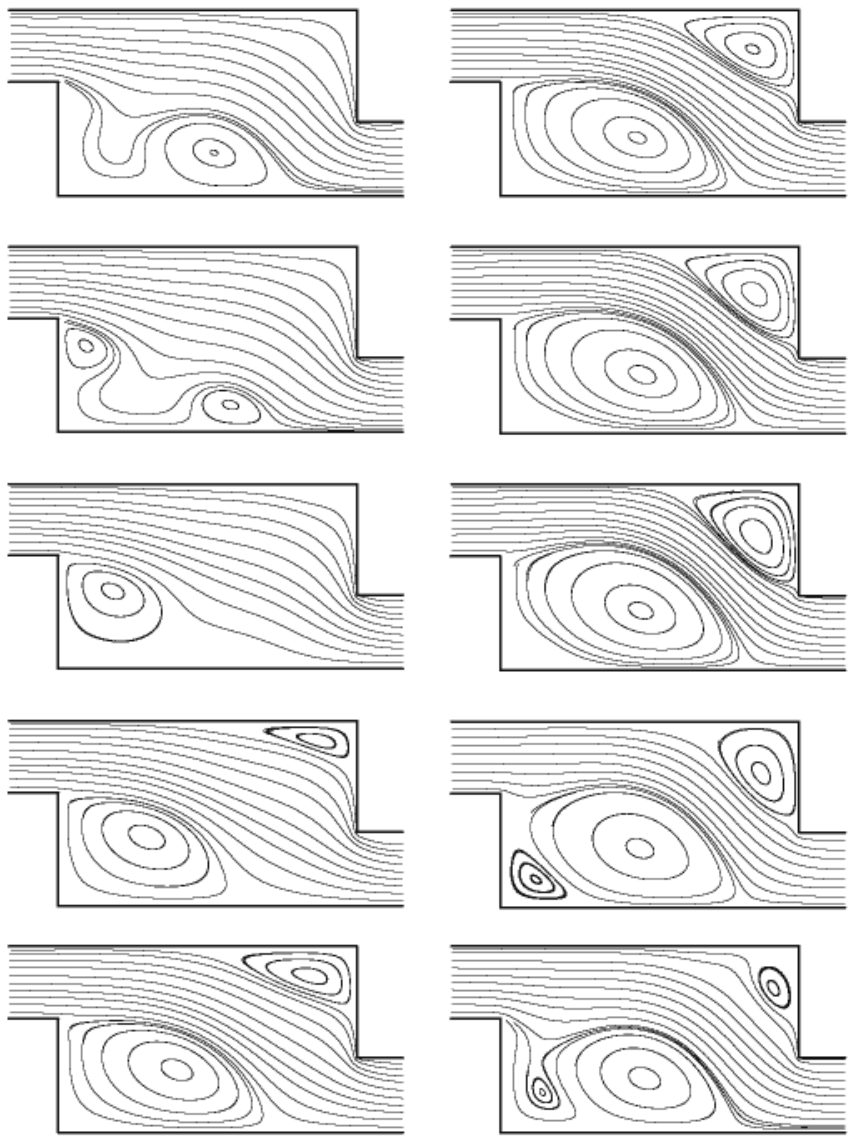

Fig. 5. Evenly time spaced series of streamlines'snapshots along a pulsation cycle for the case: $B=0.75 A, C=4.0 A, \omega=0.2$. Time reads top to bottom and left to right.

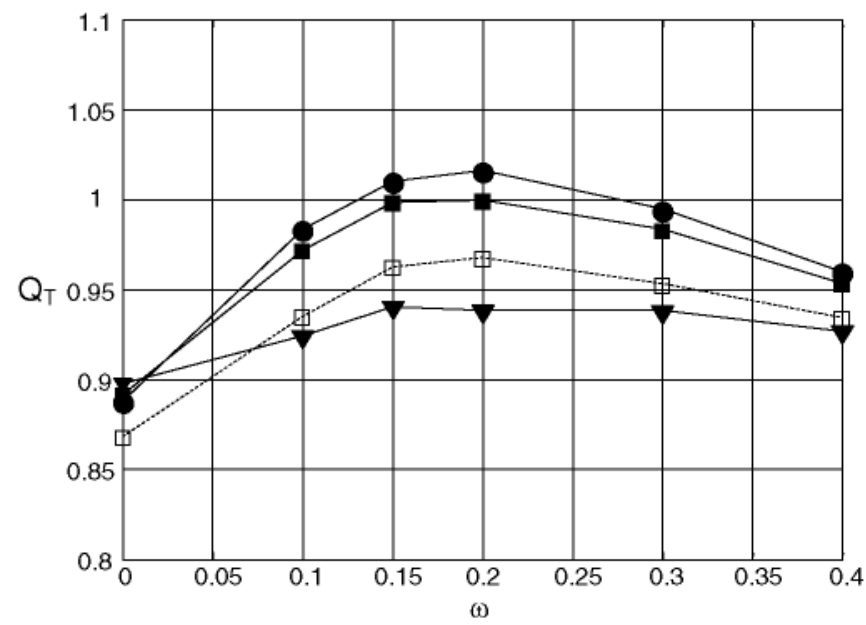

Fig. 6. Normalised total heat transfer as a function of the dimensionless pulsation frequency considering real fluid properties: $\boldsymbol{\nabla} B=0.5 A, \boldsymbol{\nabla} B=1.0 A, \bullet B=1.5 \mathrm{~A}$, and $\square$ $B=1.0 \mathrm{~A}$ with constant fluid viscosity and thermal conductivity. $C=4.0 \mathrm{~A}$ for all cases.

ties. Quantitatively speaking, the actual level of heat transfer recovery is different because the local Reynolds number is different in the vicinity of the heated walls. Figs. $6 a$ and 3 suggest that heat transfer in the wall portions $\mathrm{c}-\mathrm{d}$ and $\mathrm{g}-\mathrm{i}$ is larger that in the corresponding straight channel baseline case. This normalised heat transfer $Q_{D}$ has been plotted in Fig. 7 for the case $B=1.5 A$. In this figure, it could be observed that pulsation affects the upper (c-d) 


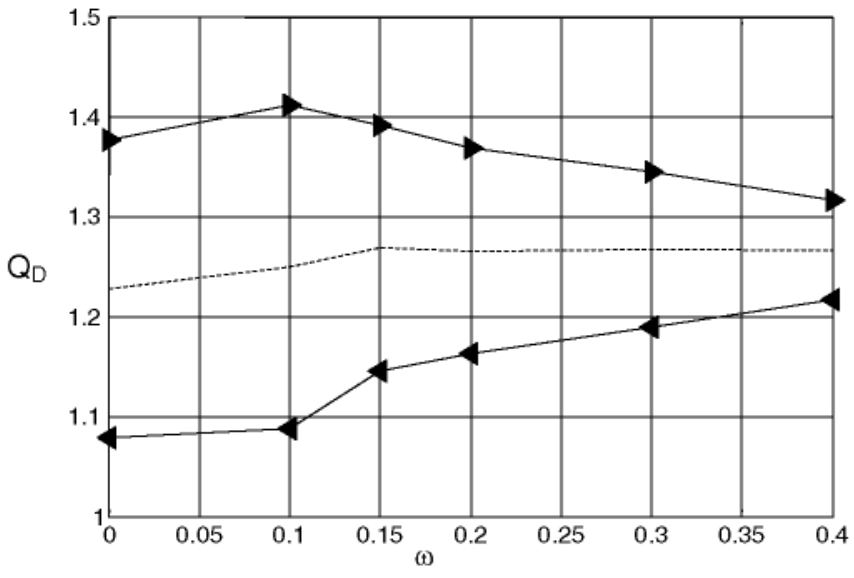

Fig. 7. Normalised heat transfer in the additional wall portions $\mathrm{c}-\mathrm{d}$ and $\mathrm{g}-\mathrm{i}$. $>$ lower portion $\mathrm{g}-\mathrm{i}$, and $\boldsymbol{4}$ upper portion $\mathrm{c}-\mathrm{d}$.

and lower $(\mathrm{g}-\mathrm{i})$ walls in a different way. Heat transfer level in the steady situation for the lower wall is $38 \%$ higher than in the baseline straight channel case. This heat transfer grows up to the $41 \%$ level at $\omega=0.1$ and, then, it decreases when increasing frequency. However, heat transfer in upper wall keeps growing along with the pulsation frequency. The net effect of flow pulsation and flow distortion at the entrance section c-g of the outlet channel is that overall heat transfer (dashed line in Fig. 7 ) is $25 \%$ higher than in the straight channel case.

We have also computed the corresponding series of cases with $C=2 A$ and the results are presented in Figs. 8 and 9 . The tendency is similar to that of cases having $C=4 A$, although there is a distinctive effect associated to the shortening of the cavity length. When considering normalised heat transfer inside the cavity (Fig. 8) it could be observed that it increases along with the pulsation frequency. However, in the case with $B=0.75 A$ the resonance region is flatter than in the case with $C=4 A$. In the remaining two cases ( $B=0.50 A$ and $B=0.25 A$ ) the heat transfer still increases at the maximum frequency $\omega=0.4$ that we have considered. When heat transfer is analysed including wall segments c-d and g-i (Fig. 9) it is found that the pulsation allows, again, for recovering the original heat transfer level associated to the straight channel. Furthermore, in this case we have observed that a slight increase of $5 \%$ above the reference level is obtained.

At this point, one of the questions that arise is why pulsation sometimes affects heat transfer and sometimes it does not. To

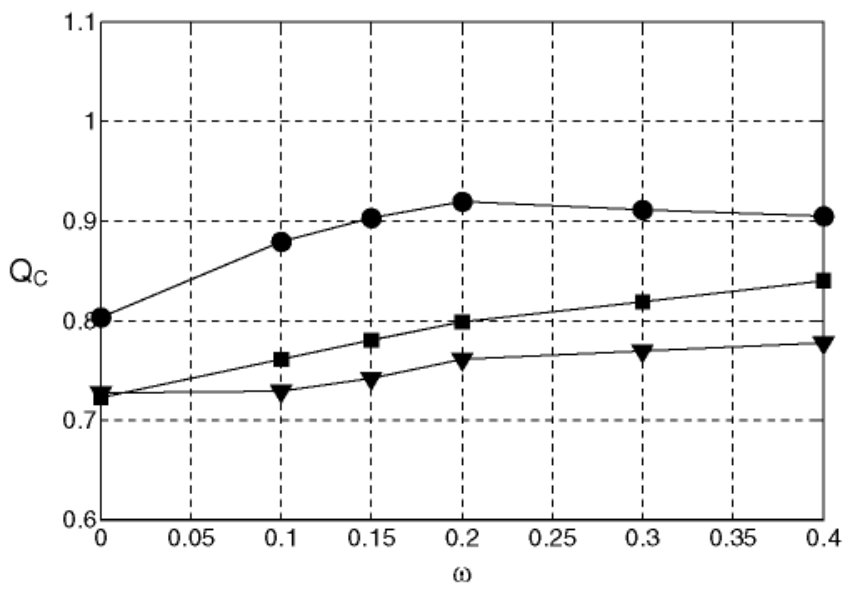

Fig. 8. Normalised cavity heat transfer as a function of the dimensionless pulsation frequency. $\nabla B=0.5 A,-B=1.0 A$, and $B=1.5 A, C=2.0 A$.

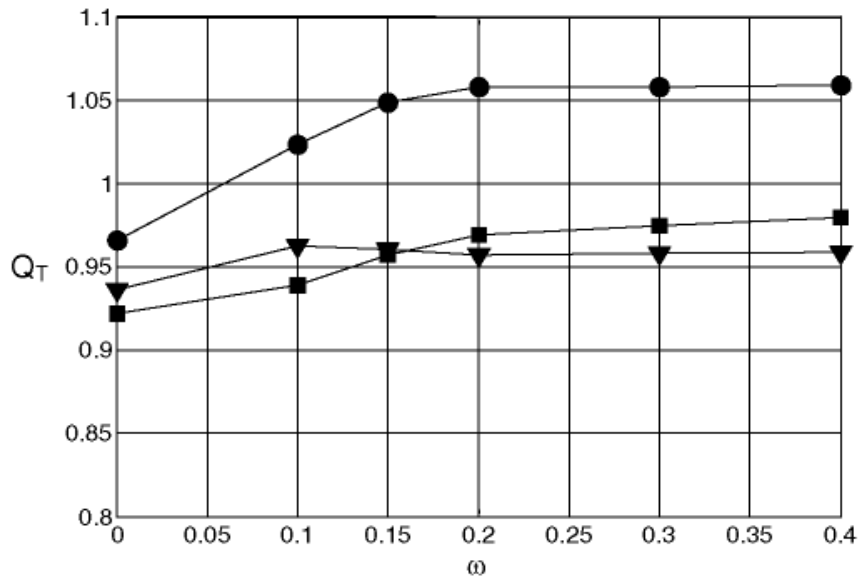

Fig. 9. Normalised total heat transfer as a function of the dimensionless pulsation frequency. $\boldsymbol{\nabla} B=0.5 A, \boldsymbol{\nabla} B=1.0 A$, and $B=1.5 A, C=2.0 A$.

summarise the results that we have obtained so far: (a) we have previously found (reference [18]) that pulsations enhances heat transfer downstream of a backwards facing step, (b) in the work presented in this paper we observe that pulsation enhances heat transfer inside a cavity, and (c) in the work presented in this paper we observe that pulsation does not exert influence when a straight channel is considered (in agreement with the results of references $[25,26])$. To try to gather additional information on the subject, we have performed an additional series of unsteady computations on a forward facing step. We have considered the basic geometry and computational domain used in reference [18]. In this case, all walls are adiabatic except in a portion downstream of the forward facing step. We computed the case with $R e=100$ at different pulsation frequencies in the range $\omega=0.0-0.4$ and found that the time averaged heat transfer is equal (within an error smaller than $1 \%$ ) to the heat transfer in the steady case. In all these cases, the pattern of streamlines in the vicinity of the heated wall regions remained constant all along the pulsation cycle. Fig. 10 shows the streamline pattern at two different times of the pulsation cycle for $\omega=0.4$. A very small recirculation region was observed right downstream of the step (where the wall was heated) but its length was of the order of one unit distance between grid points, and it did not signif-

Flow direction
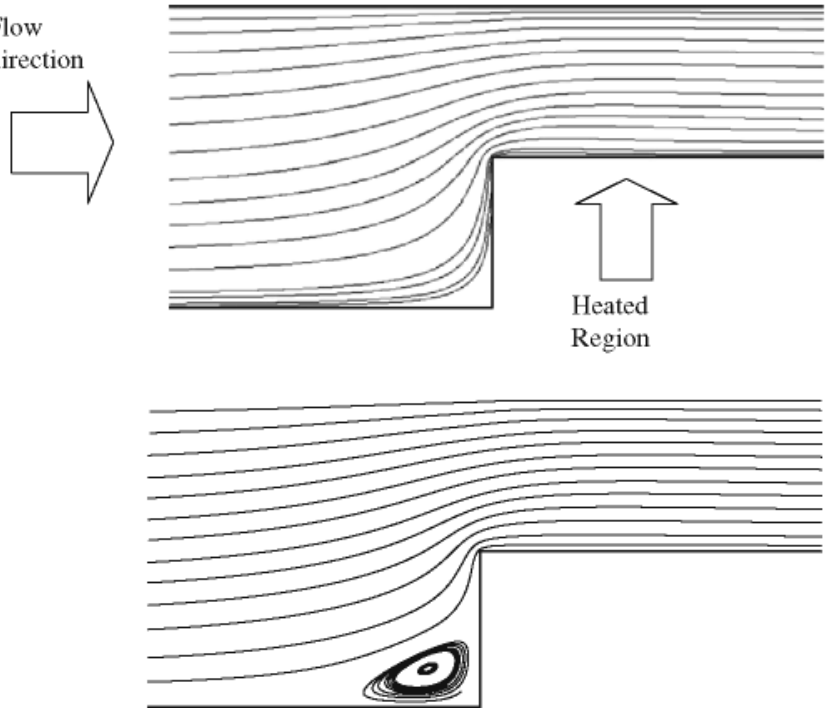

Fig. 10. Two snapshots of the streamlines' field along a pulsating cycle for the forward facing step case at $\omega=0.4$. 
icantly affect heat transfer. Therefore, it seems that for pulsation to take effect it is needed that the flow topology (streamlines topology) in the vicinity of the heated wall regions changes over time. This kind of evidence has also been provided by Chung and Tucker [27] in their study of laminar flow around a U-turn where a self-sustained separated oscillatory motion that promotes heat transfer is generated by placing a fin close to the turn. However, what we have just discussed is no proof at all and it is clear that the subject needs further investigated. For example, we have found out that heat transfer is also modified in the lower wall downstream of the outlet port (see Fig. 7 and section g-i in Fig. 1) where the streamlines pattern does not change significantly (see Fig. 5).

Finally, the issue of the sensitivity of the results obtained with regard to the computational parameters has been addressed and the results are given in Appendix A.

\section{Conclusions}

First, we have shown that, as expected, steady-state laminar convective heat transfer in a rectangular heated cavity degrades as a function of the aspect ratio between cavity height and inlet port height. This degradation is associated, basically, to the large recirculation regions that appear in the flow field, in contrast to the equivalent straight channel topology that presents the highest heat transfer rate.

We found that pulsation of the inlet flow allows for a recovery of heat transfer rate inside the cavity. The extent of this recovery depends on both the pulsation frequency and amplitude of the pressure gradient. In particular, we found that in the case when the cavity aspect ratio (length versus height) is in the range from 2.66 to 1.60 the time averaged heat transfer shows a resonant behaviour characterised by the fact that there is a specific frequency that causes the heat transfer to reach its maximum value. In fact, further increments of this resonant frequency tend to decrease the heat transfer rate. In the case of shorter cavities, with aspect ratios in the range from 1.33 to 0.80 , the behaviour is qualitatively similar although it appears that, in some cases, the resonant frequency lies beyond the range that we have considered. Also, we have found out that the presence of the cavity has the additional effect of improving heat transfer downstream of the outlet port. On the average, this improvement is of the order of $25 \%$ when compared to the reference straight channel. Regarding its dependency on frequency it shows an asymptotic behaviour; that is, no additional increments are gained for dimensionless pulsation frequencies above 0.15 .

When selecting the appropriate pulsation frequency, heat transfer in the cavity could be recovered up to the $88 \%$ level of the straight channel. If heat transfer is also measured downstream of the outlet port in a distance equal to that of the cavity, the total heat transfer (cavity plus additional section) is equal to that of the equivalent straight channel.

Summarising, we found that pulsation does not affect heat transfer in the cases of a straight channel and a forward facing step. However, pulsation does indeed affect heat transfer in the cases of a cavity and of a backwards facing step. One possible explanation is that for pulsation to exert an influence it is necessary that the topology of the streamlines in the vicinity of the heat transfer region changes over time. In the cases of the straight channel and the forward step, flow velocity changes its time-dependent level but streamlines do not change. In the cases of the cavity and the back step, the streamline pattern changes significantly over time. This conclusion is in partial agreement with other results presented in the specialised literature. However, this is no proof, and it seems clear to us that the subject needs to be further investigated in more detail. Specially, because we also found out that
Table 1

Results of the sensitivity analysis of the solutions obtained as a function of time step, artificial viscosity parameters and artificial compressibility parameter

\begin{tabular}{lllllll}
\hline Case & $\mathrm{d} t$ & $v_{2}$ & $v_{4}$ & $\beta$ & $Q_{\text {cavity }}$ & Q $_{\text {total }}$ \\
\hline S_ref & $2.50 \mathrm{e}-4$ & $1.00 \mathrm{e}-3$ & $4.00 \mathrm{e}-3$ & 200 & 0.78 & 0.96 \\
S_01 & $2.50 \mathrm{e}-4$ & $1.00 \mathrm{e}-3$ & $4.00 \mathrm{e}-3$ & 225 & 0.78 & 0.97 \\
S_02 & $2.50 \mathrm{e}-4$ & $1.00 \mathrm{e}-3$ & $4.00 \mathrm{e}-3$ & 250 & 0.79 & 0.97 \\
S_03 & $1.25 \mathrm{e}-4$ & $5.00 \mathrm{e}-4$ & $2.00 \mathrm{e}-3$ & 200 & 0.79 & 0.97 \\
S_04 & $6.25 \mathrm{e}-5$ & $2.50 \mathrm{e}-4$ & $1.00 \mathrm{e}-3$ & 200 & 0.79 & 0.97 \\
S_05 & $6.25 \mathrm{e}-5$ & $5.00 \mathrm{e}-4$ & $2.00 \mathrm{e}-3$ & 200 & 0.77 & 0.95 \\
S_06 & $2.50 \mathrm{e}-4$ & $6.67 \mathrm{e}-4$ & $2.67 \mathrm{e}-3$ & 200 & 0.79 & 0.97 \\
S_07 & $2.50 \mathrm{e}-4$ & $5.00 \mathrm{e}-4$ & $2.00 \mathrm{e}-3$ & 200 & 0.79 & 0.97 \\
S_08 & $2.50 \mathrm{e}-4$ & $2.50 \mathrm{e}-4$ & $1.00 \mathrm{e}-4$ & 200 & 0.79 & 0.97 \\
\hline
\end{tabular}

heat transfer is modified in the lower wall downstream of the outlet port where streamlines do not change significantly.

\section{Acknowledgements}

This work has been funded by the Spanish Ministry of Science and Education (Ministerio de Investigacion y Ciencia) under Plan Nacional de I+D+i contract DPI-2005-05572. The authors gratefully acknowledge this support.

\section{Appendix A}

Finally, we address the issue of the sensitivity of the results that have been presented with regard to different computational parameters. To this end, we have performed an additional series of computations where the following parameters have been changed: grid spacing, time step, artificial compressibility parameter and artificial viscosity terms. First, we have generated a new computational domain for the case $B=A$ and $C=4.0 A$. For this new computational domain we also used a Cartesian grid but, now, the spacing between points was 0.0125 instead of 0.02 that was used for the reference case. The number of total points for this new grid was 62,241 while in the reference case we used 24,651 points. We computed the most critical pulsation frequency from the computational standpoint $(\omega=0.4)$ and we obtained normalised values of the heat transfer in the cavity and in the cavity plus the portions $\mathrm{g}-\mathrm{i}$ and $\mathrm{c}-\mathrm{d}$ (see Fig. 1 ) of 0.80 and 0.97 , respectively. The values obtained with the reference grid (see Figs. 3 and 4) were 0.78 and 0.95 , respectively. That is, differences were of order of $2 \%$ so we could consider our computations as grid converged. Regarding the sensitivity study on the other computational parameters, we have selected as reference case (S_ref in Table 1) the combination of parameters that yield the largest recirculation regions ( $B=1.5 A, C=4.0 A)$ at the highest pulsation frequency $\omega=0.4$. The definition of the different cases and the results obtained are presented in Table 1. It is to be noted in this table that when we decrease the time step we also decrease the actual value of the dissipation terms. The reason is that, in our discretization scheme [18], dividing the time step by a certain factor causes, implicitly, the viscosity terms to go up by the same factor. The results presented in Table 1 show that the computations could be considered as converged with regard to time step, artificial compressibility parameter and numerical diffusion terms. Changes of the order of $400 \%$ in some of these parameters caused the computational results to change by a actor of less than $2 \%$ when compared to the reference case.

\section{References}

[1] D. Angeli, P. Levoni, G.S. Barozzi, Numerical predictions for stable buoyant regimes within a square cavity containing a heated horizontal cylinder, Int. ]. Heat Mass Transfer 51 (2008) 533-565. 
[2] B.S. Kim, D.S. Lee, M.Y. Ha, H.S. Yoon, A numerical study of natural convection in a square enclosure with a circular cylinder at different vertical locations, lnt. J. Heat Mass Transfer 51 (2008) 1888-1906.

[3] M.Y. Ha, I.K. Kim, H.S. Yoon, S. Lee, Unsteady fluid flow and temperature fields ina horizontal enclosure with an adiabatic body. Plyss. Fluids 14(2002) 3189-3202.

14] E.V. Kalabin, M.V. Kanashina, P.T. Zublkov, Heat transfer from the cold wall of a square cavity to the hot one by oscillatory natural convection, Numer. Heat Transfer A 47 (2005) 609-619.

[5] S.K. Mahapatra, Anjan Sarkar, A. Sarkar, Numerical simulation of opposing mixed convection in differentially heated square enclosure with partition, Int. J. Therm. $5 c i .46$ (2007) 970-979.

[6] G. Guo, MA.R. Sharif, Mixed convection in rectangular cavities at various aspect ratios with moving isothermal sidewalls and constant flux heat source on the bottom wall, Int. J. Therm. Sci. 42 (2004) 465-475.

[7] S.M. Saeidi, J.M. Khodadadi, Forced convection in a square cavity with inlet and outlet ports. Int. J. Heat Mass Transfer 49 (2006) 1896-1906.

[8] Y.S. Leung Ki, D. Maillefer, G. Rey-Mermet, P.A. Monkewitz, P. Renaud, H.T.G. Van lintel, Microfluidics of a planar microfabricated fluid filter. Micro-ElectroMechanical Systems - 1998. ASME DSC vol. 66 (1998) 165-170.

[9] C. Lee, E.H. Yang. S.M. Saeidi, J.M. Khodadadi, Fabrication, characterization and computational modeling of a piezoelectrically actuated microvalve for liquid flow control, J. Microelectromech. Syst. 15 (2006) 686-696.

|10| S.M. Saeidi. J.M. Khodadadi, Transient flow and heat transfer leading toperiodic state in a cavity with inlet and outlet ports due to incoming flow oscillation, Int. J. Heat Mass Transfer 50 (2007) 530-538.

|11| S.Z. Shuja, B.S. Yilbas, M.O. Iqbal, Mixed convection in a square cavity due to heat generating rectangular body, effect of cavity exit port location, Int J. Numer. Meth. Heat Fluid Flow 10 (2000) 824-841.

|12| S. Singh, M.A.R. Sharif, Mixed convective cooling of a rectangular cavity with inlet and exit openings on differentially heated side walls, Numer. Heat Transfer A 44 (2003) 233-253.

[13] P. Verboven, A.K. Datta, N.T. Ahn, N. Scheerlinck, B.M. Nicolai, Computation of airflow effects on heat and mass transfer in a microwave oven, J. Food Eng. 59 (2003) 181-190

[14] Y. Masuda, T. Aizawa, M. Kanakubo, N. Saito, Y. Ikushima, Numerical simulation of two dimensional piston effect and natural convection in a square cavity heated from one side. Int. Commun. Heat Mass Transfer 31 (2004) 151-160.

[15] 5.H. Tasnim, M.R. Collins, Numerical analysis of heat transfer in a squale cavity with a baffle on the hot wall, Int. Commun. Heat Mass Transfer 31 (2004) 639650.

[16] Y. Lin, B. Farouk, E.5. Oran, Interactions of thermally induced acoustic waves with buoyancy induced flows in rectangular enclosures, Int. J. Heat Mass Transfer 51 (2008) 1665-1674.

[17] F.P. Incropera, D.P. DeWitt, Introduction to Heat Transfer, Wiley. New York 1996. p. 764 (Table A-6).

[18] A. Velázquez, J.R. Arias, B. Méndez, laminar heat transfer enhancement downstream of a backward facing step by using a pulsating flow, Int. J. Heat Mass Transfer 51 (2008) 2075-2089.

[19] D. Das, J.H. Arakeri. Transition of unsteady velocity profiles with reverse flow. J. Fluid Mech. 374 (1998) 251-283.

[20] R. Alkavan, R.D. Kanm, A.H. Shapiro, An investigation of transition to tubulence in bounded oscillatory Stokes flows. Part 1. Experiments, J. Fluid Mecl. 225 (1991) 395-422.

[21] R. Alkhavan, R.D. Kanm, A.H. Shapiro, An investigation of transition to turbulence in bounded oscillatory Stokes flows. Part 2. Numerical simulations, J. Fluid Mech. 225 (1991) 423-444.

[22] M. Hino, M. Sawamoto, S. Takasu, Experiments on transition to tubulence in an oscillatory pipe flow, J. Fluid Mech. 75 (1976) 193-207.

[23] B. Mendez, A. Velazquez. Finite point solver for the simulation of 2-D laminar incompressible unsteady flows, Comput. Meth. Appl. Mech. Eng. 193 (2004) 825-848.

[24] J.C. Tannehill, D.A. Anderson, R.H. Fletcher, Computational Fluid Mechanics and Heat Transfer, Taylor \& Francis, Philadelphia, PA, 1997.

[25] J.C. Yu, Z,X. li, T.S. Zhao, An analytical study of pulsating laminar heat convection in a circular tube with constant heat flux, lnt. J. Heat Mass Transfer 47 (2004) 5297-5301.

[26] H. Chattopadhyay, F. Durst, S. Ray, Analysis of heat transfer in simultaneously developing pulsating laminar flow in a pipe with constant wall temperature. Int. Commun. Heat Mass Transfer 33 (2006) 475-481.

[27] Y.M. Chung, P.G. Tucker, Numerical studies of heat transfer enhancements in laminar separated flows, Int. J. Heat Fluid Flow 25 (2004) 22-31. 